\title{
Faster Hash-Based Signatures with Bounded Leakage
}

\author{
Thomas Eisenbarth ${ }^{1}$, Ingo von Maurich ${ }^{2(凶)}$, and Xin $\mathrm{Ye}^{1}$ \\ 1 Worcester Polytechnic Institute, Worcester, MA, USA \\ 2 Horst Görtz Institute for IT-Security, Ruhr-University Bochum, Bochum, Germany \\ \{teisenbarth, xye\}@wpi.edu, ingo.vonmaurich@rub.de
}

\begin{abstract}
Digital signatures have become a key component of many embedded system solutions and are facing strong security and efficiency requirements. At the same time side-channel resistance is essential for a signature scheme to be accepted in real-world applications. Based on the Merkle signature scheme and Winternitz one-time signatures we propose a signature scheme with bounded side-channel leakage that is secure in a post-quantum setting. Novel algorithmic improvements for the authentication path computation bound side-channel leakage and improve the average signature computation time by close to $50 \%$ when compared to state-of-the-art algorithms. The proposed scheme is implemented on an Intel Core i7 CPU and an AVR ATxmega microcontroller with carefully optimized versions for the respective target platform. The theoretical algorithmic improvements are verified in the implementations and cryptographic hardware accelerators are used to achieve competitive performance.
\end{abstract}

Keywords: Hash-based cryptography $\cdot$ Signatures $\cdot$ Side-channel leakage $\cdot$ Software $\cdot$ Microcontroller $\cdot$ Post-quantum cryptography

\section{Motivation}

With the increasing popularity of contactless smart cards and near field communication, digital signature engines have become a key component of many embedded system solutions. The applications of digital signatures are numerous, ranging from identification over electronic payments to firmware updates and protection against product counterfeiting. Due to the high computational requirements for public-key cryptography, providing efficient signatures on embedded microprocessors without dedicated co-processors is a challenge. At the same time, side channel attacks are considered a serious threat for such embedded implementations. On the downside, adding effective protection against attacks like power or EM analysis is costly in terms of space and computation time. Hence, side-channel resistant public key engines are often just too bulky for widespread adoption. Exploring public key schemes that are both efficient on embedded platforms and offer inherent side-channel resistance can be a superior alternative to the prevailing choices of (EC-)DSA and RSA. 
New research directions in theoretical cryptography, namely leakage resilient cryptographic schemes, suggest that performing cryptographic algorithms in a different way might make them inherently resistant against side-channel attacks without the need of further implementational countermeasures. Instead of protecting a key that is used over and over again, these schemes limit the leakage that an attacker can observe for a given key (or state) by limiting the number of accesses to it. The groundbreaking work of Faust et al. [9] shows a scheme that provides choosable many leakage resilient signatures. The approach builds on a signature scheme that only leaks an admissible amount of information when executed up to three times. The scheme does not explicitly propose or recommend an underlying signature scheme. But when instantiated with one of the prevailing signature schemes, the leakage resilient signature engine becomes practically infeasible: each generated leakage-resilient signature requires three signature generations and two key generations of the underlying signature.

Prior work by Rohde et al. [22] as well as by Hülsing et al. [11] suggest that the Merkle Signature Scheme (MSS) in combination with Winternitz One-Time Signatures (W-OTS) is a possible choice for a time-limited signature scheme and can be efficiently implemented in embedded systems. We analyze and extend the proposal by Rohde et al. and propose several modifications that lead to significant performance improvements and bounded side-channel leakage. One of the key components of the analyzed MSS engine is the Pseudo Random Number Generator (PRNG) used to generate the private signing key. The PRNG is a self-contained component and is desired to be leakage resilient. Another building block for the one-time signatures is a one-way function that needs to have bounded leakage. Other parts of the engine, such as a collision resistant hash function needed for the Merkle tree only process public knowledge and are thus leakage-agnostic.

Contribution. Compared to the state-of-the-art, the proposed scheme provides bounded leakage at comparable cost to an unprotected ECC engine, which enables and encourages a wide deployment. We implement the proposed signature scheme on two wide-spread platforms (Intel Core i7 CPU and low-cost AVR 8-bit microcontroller) targeting a security level of 80-bit and making use of available cryptographic hardware accelerators to gain maximum efficiency. Furthermore, we propose an improved algorithm for the authentication path computation of a Merkle tree which limits side-channel leakage when signature keys are generated using a secure PRNG. At the same time we decrease the average computation time by close to $50 \%$ compared to the most efficient authentication path computation algorithm at the price of a slightly increased memory consumption. Explicit formulas are developed to quantify the amount each leaf of the Merkle tree is computed during the authentication path computation. The drawback of current authentication path computation algorithms is the unbalanced number of computations per leaf. Our improved algorithm mitigates this issue by reducing the number of computations for often used leaves and allows for more efficient computation of the authentication path. 


\section{Hash-Based Signatures}

In the following we describe the foundations of the Merkle signature scheme. It was introduced in [19] and a detailed description of MSS can be found in [6]. Details about the implementation inspiring our work are given in [22]. We use Winternitz one-time signatures [8] for message signing. The one-time keys are generated using a PRNG to minimize storage requirements as proposed in [22].

The following components use an at least second preimage resistant, undetectable $n$-bit one-way function $f$ and a cryptographic $m$-bit hash function $g$ :

$$
f:\{0,1\}^{n} \rightarrow\{0,1\}^{n}, g:\{0,1\}^{*} \rightarrow\{0,1\}^{m}
$$

\subsection{The Merkle Signature Scheme}

Given a One-Time Signature Scheme (OTSS) a tree height $H$ is chosen to allow for the creation of $2^{H}$ signatures that are verifiable with the same verification key. Let the nodes of the Merkle tree be denoted as $\nu_{h}[s]$ with $h \in\{0, \ldots, H\}$ being the height of the node and $s \in\left\{0, \ldots, 2^{H-h}-1\right\}$ being the node index on height $h$.

Key Generation. The $2^{H}$ leaves of the Merkle tree are defined to be digests $g\left(Y_{i}\right)$ of one-time verification keys $Y_{i}$. Starting from the leaves, the MSS verification key which is the root node of the Merkle tree $\nu_{H}[0]$ is generated following

$$
\nu_{h+1}[i]=g\left(\nu_{h}[2 i] \| \nu_{h}[2 i+1]\right), \quad 0 \leq h<H, \quad 0 \leq i<2^{H-h-1},
$$

meaning that a parent node is generated by hashing the concatenation of its two child nodes.

Signature Generation. A Merkle signature $\sigma_{s}(d)$ of a digest $d=g(M)$ of a message $M$ consists of a signature index $s$, a one-time signature $\sigma_{\mathrm{OTS}}$, a onetime verification key $Y_{s}$, and an authentication path $\left(\mathrm{AUTH}_{0}, \ldots, \mathrm{AUTH}_{H-1}\right)$ that allows the verification of the one-time signature with respect to the public MSS verification key, hence

$$
\sigma_{s}(d)=\left(s, \sigma_{\mathrm{OTS}}, Y_{s},\left(\mathrm{AuTH}_{0}, \ldots, \mathrm{AuTH}_{H-1}\right)\right) .
$$

The signature index $s \in\left\{0, \ldots, 2^{H}-1\right\}$ is incremented with every issued signature. The OTSS is applied using signature key $X_{s}$ to generate the signature $\sigma_{\text {OTS }}=\operatorname{Sign}_{\mathrm{OTS}}\left(d, X_{s}\right)$ of the message digest $d$. The authentication path for the $s$ th leaf are all sibling nodes $\mathrm{AUTH}_{h}, h \in\{0, \ldots, H-1\}$ on the path from leaf $\nu_{0}[s]$ to the root node $\nu_{H}[0]$. It enables the verifier to recompute the root node of the Merkle tree and authenticates the current one-time signature.

We would like to stress that the signature generation reflects the structure of an online/offline signature scheme. The authentication path only depends on the OTSS verification key $Y_{s}$ which is known prior to the message and hence can be precomputed. 
Signature Verification. Given a message digest $d=g(M)$ and a signature $\sigma_{s}(d)$ the verifier checks the one-time signature $\sigma_{\text {OTS }}$ with the underlying onetime signature verification algorithm Verify $_{\text {OTS }}\left(d, \sigma_{s}(d)\right)$. In addition, the root node is reconstructed using the provided authentication path $\phi_{h+1}=\left\{\begin{array}{l}g\left(\phi_{h} \| \mathrm{AUTH}_{h}\right), \text { if }\left\lfloor s / 2^{h}\right\rfloor \equiv 0 \bmod 2 \\ g\left(\mathrm{AuTH}_{h} \| \phi_{h}\right), \text { if }\left\lfloor s / 2^{h}\right\rfloor \equiv 1 \bmod 2\end{array}, \quad \phi_{0}=\nu_{0}[s], h=0, \ldots, H-1\right.$.

If the one-time signature $\sigma_{\text {OTS }}$ is successfully verified and $\phi_{H}$ is equal to $\nu_{H}[0]$ the MSS signature is accepted.

\subsection{Winternitz One-Time Signatures}

Winternitz OTS [8] are a convenient choice for the one-time signature scheme, as they reduce the overall signature length. The Winternitz parameter $w \geq 2$ determines how many bits are signed simultaneously and $t$ determines of how many random $n$-bit strings $x_{i}$ the Winternitz signature keys consist.

$$
t=t_{1}+t_{2}, t_{1}=\left\lceil\frac{n}{w}\right\rceil, t_{2}=\left\lceil\frac{\left\lfloor\log _{2} t_{1}\right\rfloor+1+w}{w}\right\rceil
$$

Key Generation. A W-OTS signature key $X=\left(x_{0}, \ldots, x_{t-1}\right)$ is generated by selecting $t$ random bit strings $x_{i} \in\{0,1\}^{n}, 0 \leq i<t$. The W-OTS verification key $Y=g\left(y_{0}\|\ldots\| y_{t-1}\right)$ is computed from the signature key by applying $f$ $2^{w}-1$ times to each $x_{i}$ giving $y_{i}=f^{2^{w}-1}\left(x_{i}\right), 0 \leq i<t$ and computing the hash of the concatenated $y_{i}$ 's. Note, the superscript denotes multiple executions of $f$, e.g., $f^{2}\left(x_{i}\right)=f\left(f\left(x_{i}\right)\right)$ and $f^{0}\left(x_{i}\right)=x_{i}$.

Signature Generation. A signature for a message $M$ is created by signing its digest $d=g(M)$ under key $X$. Digest $d$ is divided into $t_{1}$ blocks $b_{0}, \ldots, b_{t_{1}-1}$ of length $w$ and a checksum $c=\sum_{i=0}^{t_{1}-1}\left(2^{w}-b_{i}\right)$ is computed. Checksum $c$ is divided into $t_{2}$ blocks $b_{t_{1}}, \ldots, b_{t-1}$ of length $w$ (zero-padding to the left is applied if $c$ or $d$ are no multiples of $w)$. The W-OTS signature $\sigma_{\mathrm{W}-\mathrm{OTS}}=\left(\sigma_{0}, \ldots, \sigma_{t-1}\right)$ is computed with $\sigma_{i}=f^{b_{i}}\left(x_{i}\right), 0 \leq i<t$.

Signature Verification. Given a message digest $d=g(M)$, a signature $\sigma_{\mathrm{W}-\mathrm{OTS}}$ and a verification key $Y_{s}$ the verifier generates blocks $b_{0}, \ldots, b_{t-1}$ from $d$ as in signature generation and reconstructs

$$
Y_{s}^{\prime}=g\left(f^{2^{w}-1-b_{0}}\left(\sigma_{0}\right)\|\ldots\| f^{2^{w}-1-b_{t-1}}\left(\sigma_{t-1}\right)\right) .
$$

If $Y_{s}^{\prime}$ equals $Y_{s}$ the signature is valid, otherwise it has to be rejected. When using W-OTS signatures in MSS, transmitting $Y_{s}$ and comparing $Y_{s}$ to $Y_{s}^{\prime}$ can be omitted. $Y_{s}^{\prime}$ can simply be used together with the nodes of the authentication path to recompute the root of the Merkle tree. If the recomputed root equals the MSS public key, then $Y_{s}^{\prime}$ is a valid OTS verification key. 


\subsection{Private Key Generation}

Storing $2^{H}$ one-time signature or verification keys can be an infeasible task, especially on constrained implementation platforms. Generating keys on-the-fly by using a PRNG significantly reduces the required storage space (cf. [22]).

Each W-OTS signature key $X_{i}=\left(x_{0}, \ldots, x_{t-1}\right), 0 \leq i<2^{H}$ is generated by the PRNG from a seed SEEDW-OTS $i$. These seeds in turn are also generated by the PRNG from a initial randomly selected seed $\operatorname{SEED}_{0} \in_{R}\{0,1\}^{n}$ which serves as the MSS signature key. On input of $k_{\mathrm{i}}$ the PRNG outputs a random string $r_{i+1}$ and an updated seed $k_{i+1}$.

$$
\text { PRNG }:\{0,1\}^{n} \rightarrow\{0,1\}^{n} \times\{0,1\}^{n}, k_{i} \rightarrow\left(k_{i+1}, r_{i+1}\right)
$$

Starting from the initial $\mathrm{SEED}_{0}$ the seeds for the signature keys $\mathrm{SEEDW}_{\mathrm{W}-\mathrm{OTS}}$ are created by

$$
\left(\operatorname{SeEd}_{i+1}, \operatorname{SeEDh-Ots}_{i}\right) \leftarrow \operatorname{Prng}\left(\operatorname{SeEd}_{i}\right), 0 \leq i<2^{H} .
$$

The $t n$-bit strings of the $i$-th W-OTS signature key $X_{i}=\left(x_{0}, \ldots, x_{t-1}\right), 0 \leq$ $i<2^{H}$ are then generated by

$$
\left.\left(\mathrm{SEEDW-OTS}_{i}, x_{j}\right) \leftarrow \mathrm{PRNG}_{\left(\mathrm{SEEDW}_{\mathrm{W}-\mathrm{OtS}}\right)}\right), 0 \leq j<t .
$$

\subsection{Authentication Path Computation}

Creating an authentication path for a specific leaf $s$ can be accomplished by storing all tree nodes in memory and looking up the required nodes when needed. However, because of the exponential growth of nodes in tree height $H$ this approach becomes infeasible for reasonable practical applications. Hence, algorithms for efficient on-the-fly authentication path computation during signature generation are required.

The currently best known algorithm for on-the-fly computation of authentication nodes is the BDS algorithm [6] (Algorithm 3, cf. Appendix). It makes use of several treehash algorithm instances $\mathrm{TrEEHASH}_{h}$ for heights $0 \leq h \leq H-K-1$. The treehash algorithm was introduced in [19] and modified in [25]. It allows to efficiently create (parts of) Merkle trees. In the BDS algorithm each instance is initialized with a leaf index $s$ to which it computes the corresponding node value. Each instance is updated until the required authentication node is computed. During a treehash update the next leaf is created and parent nodes are computed if possible.

The generation of the authentication path is split up into two parts that go alongside with the key and signature generation of MSS. During key generation all treehash instances TrEeHASH ${ }_{h}$ are initialized with $\nu_{h}[3]$ and the first authentication path stored is $\mathrm{AUTH}_{h}=\nu_{h}[1], 0 \leq h \leq H-1$.

The BDS algorithm generates left authentication nodes either by computing the leaf value or by one hash-function evaluation of the concatenation of two previously computed nodes that are held in memory. Right authentication 
nodes in contrast are computed from the leaf up, which is computationally more expensive. Since right nodes close to the top are expensive to compute a positive integer $K \geq 2,(H-K$ even $)$ decides how many of these nodes are stored in $\operatorname{RETAIN}_{h}, H-K \leq h \leq H-2$ during key generation.

Authentication nodes change every $2^{h}$ steps for height $h$. During signature generation the treehash instances are updated and if a authentication node from a treehash instance is used, the instance is re-initialized to compute the next authentication node for that height.

\subsection{Security of MSS}

The security properties of the signature scheme described above is discussed in [6]. Specifically, the work shows that the Lamport-Diffie one-time signatures [15] are existentially unforgeable under an adaptive chosen message attack (i.e., CMA-secure), if the chosen one-way function is preimage resistant. The employed Merkle signature scheme is also CMA-secure if the underlying OTS is CMA-secure and if the underlying hash function is collision resistant. For increased efficiency (and shorter signatures) we chose Winternitz OTS rather than the classic Lamport-Diffie OTS. The security of the Winternitz one-time signatures is discussed in $[4,8,10]$. The findings in [4] and [10] show that Winternitz OTS are CMA-secure if used with pseudo-random functions or collisionresistant, undetectable one-way functions, respectively. The level of bit security lost by using a small Winternitz-parameter is in both cases rather small. In our case, the biggest Winternitz parameter is $w=4$, hence we still provide a security level of approx. 95 bits for a 128-bit PRF or 116 bits for W-OTS+ [10]). Related discussions for a similar MSS scheme can also be found in [5].

\subsection{Bounded Leakage for MSS}

The presented design has several features that bound leakage of secret information. First, the design consists of many one-time signatures with independent keys. This means there is no key reuse, and hence leakage of one OTS key does not reveal information about the other keys. Major parts of the performed computations are in the Merkle tree. Since the Merkle tree is public, computations within the tree do not leak any secret information. Hence, leakage of $g$ is not an issue.

Secret information is only processed during signing and key generation. Key generation usually takes place in a secure environment, as key generation is usually too costly to be performed on the embedded system. However, even if key generation leaks, it is a single sequence of leakage for all parts of the key, i.e., all one-time keys leak exactly once. Critical information leakage can only happen during signing. If all OTS keys would be stored, they could be chosen independently and would leak exactly once, when used for signing (assuming that only computation leaks information [20]). In this case, an adversary would get, at most, two observations per key (one during key generation and one at signing), outperforming the scheme described in [9]. However, as described in Sect. 2.3, 
the OTS keys are generated on-the-fly using a PRNG to achieve a scheme suited for embedded devices. In this case each signing operation consists of three steps: (i) performing one OTS, (ii) updating the state (requires recomputation of verification keys), and (iii) computing the authentication path. Since the Merkle tree is public, no secret information is revealed during authentication path computation. The OTS itself only leaks information about the current OTS key, i.e. one additional leakage for each key. The main leakage occurs during the state updates, which result in repeated execution of the PRNG and recomputation of verification keys that leak information about the corresponding OTS key.

Each PRNG update reveals information about one OTS key and the internal state of the PRNG. As the described scheme generates several one-time keys more than once, the PRNG can be executed $l$ times on the same input, where $l$ is determined by the parameters of the BDS algorithm. That is, each $\operatorname{SEED}_{i}$ has up to $l$ leakages as PRNG input. The OTS keys $x_{i}$ are derived from an initial seed SEEDh-ots $i$ by the same PRNG. The $x_{i}$ serve as input for the one-way function $f$. That is, each $\operatorname{SeEDW-OTS}_{i}$ has up to $l$ leakages as input to PRNG; each $x_{i}$ is either known by the adversary (as part of the signature) or has up to $l$ leakages as input of $f$ during verification key recomputation and signing.

\section{Optimized Authentication Path Computation}

Since the Merkle-tree is not stored, the parts of the Merkle tree needed for the authentication path must be generated. One optimized algorithm for this purpose is the BDS algorithm [6]. Its design goal was to minimize costly leaf computations. However, to minimize the leakage, it is also important to balance leaf computations. In the following we describe further optimizations that reduce the number of computations for each individual leaf, thereby minimizing the maximum leakage per private key computation. We furthermore reduce the overall computation time by close to $50 \%$, at the cost of a slightly increased memory usage.

\subsection{Authentication Path Computation}

The authentication path consists of nodes of the Merkle tree. For the computation of upcoming authentication nodes we use several stacks of nodes for different heights of the tree. Treehash instances $\mathrm{TrEEHASH}_{h}$ are used for heights $0 \leq h \leq H-K-1$. Each instance is initialized with a leaf index $s$ and is updated in Algorithm 3 until the required authentication node is computed. During a treehash update the next leaf is created and parent nodes are computed by hashing previously created nodes if possible. Authentication nodes change every $2^{h}$ steps for height $h$ and if an authentication node is used from a treehash instance, this instance is re-initialized to compute the following authentication node for that height. 
Preliminaries. The total number of leaf computations that occur during execution of Algorithm 3 can be calculated by counting all invocations of LEAFCALC, a function that on input $s$ outputs leaf $\nu_{0}[s]$. As mentioned in [6] it is possible to omit LEAFCALC in Step 3 of Algorithm 3 since the $s$ th W-OTS key pair is used to sign the current message, hence the verification key can be computed from the signature and one additional hash computation yields leaf $\nu_{0}[s]$. If a different OTSS is used the verification key is part of the OTS and can be hashed to create $\nu_{0}[s]$. This saves $2^{H-1}$ LEAfCALC invocations. Careful analysis of Algorithm 3 leads to the total number of leaf computations in the BDS algorithm

$$
N_{H, K_{\text {total }}}=\sum_{h=0}^{H-K-1}\left(2^{H-1}-2^{h+1}\right)=(H-K) 2^{H-1}-2^{H-K+1}+2 .
$$

In order to count the necessary computations for a specific leaf $s$ during execution of Algorithm 3 we have to consider all occurrences of $s$ as parameter of LEAFCALC, except for when $s$ is a left leaf (Step 3 of Algorithm 3), as explained above. To determine if leaf $s$ is computed in treehash instance $\mathrm{TREEHASH}_{h}$ we

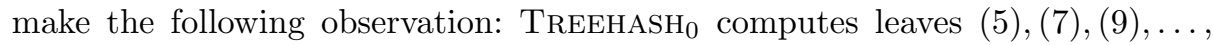
Treenash $_{1}$ computes leaves $(10,11),(14,15), \ldots$, TrEenash $_{2}$ computes leaves $(20,21,22,23),(28,29,30,31), \ldots$ and so forth. Hence, the total number of computations for leaf $s$ is given by

$$
N_{H, K}(s)=\sum_{h=0}^{H-K-1}\left\lfloor\frac{s \bmod 2^{h+1}}{2^{h}}\right\rfloor \cdot\left\lceil\frac{\left\lfloor\frac{s}{5 \cdot 2^{h}}\right\rfloor}{2^{H}}\right\rceil
$$

Drawbacks. A drawback of the BDS algorithm (Algorithm 3) is that it does not balance the computation of leaf nodes. There are leaves that are calculated various times, while others are barely touched. In terms of side-channel leakage this is undesirable. On average each leaf of the Merkle tree is computed $\overline{N_{H, K}}=$ $N_{H, K_{\text {total }}} / 2^{H} \approx \frac{1}{2}(H-K)$ times. However, the computations per leaf deviate from the average as shown in Fig. 1 for a Merkle tree $(H=10, K=2)$ with 1024 leaves.

\subsection{Balanced Authentication Path Computation}

Since the rightmost nodes of each treehash instance are calculated most frequently, we propose to cache and reuse them for balancing the leaf computations. We use an array Rightnodes to store those nodes. Note, the root of each treehash instance and the complete treehash instance TREEHASH $\mathrm{TH}_{0}$ are not stored since lower treehash instances do not require those nodes. Besides reducing the side-channel leakage for heavy duty leaves, this also leads to a significantly reduced computation time, at the cost of an increased memory consumption.

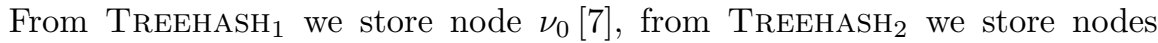
$\nu_{1}[7]$ and $\nu_{0}[15]$ and so on. More generally, we store $h$ nodes $\nu_{j}\left[2^{2+h-j}-1\right]$, 


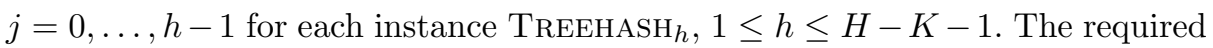
storage space is

$$
S_{\text {RightNodes }}(H, K)=\sum_{h=1}^{H-K-1} h=\left(\begin{array}{c}
H-K \\
2
\end{array}\right)=\triangle_{H-K-1} .
$$

Table 1 lists the storage requirements for common $H-K$ values. The initialization of the RightNODES array is done during the computation of the public key of the Merkle tree. The updated initial setup is formalized in Algorithm 2.

Table 1. Storage space required by the Rightnodes array where the rightmost nodes of each treehash instance TREEHASH $h, h=1, \ldots, H-K-1$ are stored for reusage by lower treehash instances.

\begin{tabular}{rrrrr}
\hline$H-K$ & $\triangle_{H-K-1}$ & 128-bit digest (byte) & 160-bit digest (byte) & 256-bit digest (byte) \\
\hline 6 & 15 & 240 & 300 & 480 \\
8 & 28 & 448 & 560 & 896 \\
10 & 45 & 720 & 900 & 1440 \\
12 & 66 & 1056 & 1320 & 2112 \\
14 & 91 & 1456 & 1820 & 2912 \\
16 & 120 & 1920 & 2400 & 3840 \\
18 & 153 & 2448 & 3060 & 4896 \\
\hline
\end{tabular}

In Step 5 of Algorithm 3 the treehash instances receive updates if they are initialized and not finished. In every update one leaf is computed and higher nodes are generated if possible by hashing concatenated nodes from the stack. During the last update before the treehash instance is finished, the rightmost leaf of this treehash instance is computed and all other rightmost nodes of this treehash instance are consecutively generated. If the leaf index $s \equiv 2^{h}-1 \bmod 2^{h}$ in instance TREEHASH $_{h}$, we store the following nodes in the RIGHTNODES array starting from offset $h(h-1) / 2$. An adapted version of the treehash update algorithm is given in Algorithm 1.

In every second re-initialization of treehash instances $\mathrm{TREEHASH}_{h}, h=0, \ldots$, $H-K-2$ the authentication node can be copied from the RightNODES array because it has been computed before by treehash instance TREEHASH $_{h+1}$. If $s+1 \equiv 0 \bmod 2^{h+2}$ the authentication node can be copied from the RIGHTNODES array and if $s+1 \equiv 2^{h+1} \bmod 2^{h+2}$ the authentication node has to be computed. If we can reuse nodes, we not only copy the authentication node (root of $\mathrm{TREEHASH}_{h}$ ) but also its rightmost child nodes from Rightnodes, so they can be reused for instances TreEHAsh $j, j<h$. This improvement can be easily integrated into the BDS algorithm by modifying Step 4c) accordingly.

Comparison. In order to quantify our improvements, we give the total amount of leaf computations and show how to determine the leaf computations for a 
specific leaf $s$. As before, each instance $\mathrm{TREEHASH}_{h}$ computes $2^{h}$ leaves until they are finished. The re-initializations however are halved for treehash instances TREEHASH $_{h}, h=0, \ldots, H-K-2$, to $2^{H-h-2}-1$ re-initializations because in half of all cases previously computed nodes can be copied from the RIGHTNODES array and the LEAFCALC computations are skipped. Hence, the number of calls

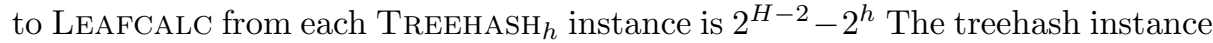
TreEHASH $_{H-K-1}$ cannot copy nodes from higher instances since it is the topmost treehash instance. It calls LEAFCALC as before, resulting in $2^{H-1}-2^{H-K}$ computations. The total number of leaf computations is

$$
\begin{aligned}
N_{H, K_{\text {total }}}^{\prime} & =\sum_{h=0}^{H-K-2}\left(2^{H-2}-2^{h}\right)+2^{H-1}-2^{H-K} \\
& =(H-K+1) 2^{H-2}-3 \cdot 2^{H-K-1}+1 .
\end{aligned}
$$

When compared to $N_{H, K_{\text {total }}}$ of the BDS algorithm this is nearly a $50 \%$ reduction.

To retrieve the number of leaf computations in the improved version for a specific leaf $s$ we have to check whether $s$ is a left or a right leaf. If $s$ is even, it is a left leaf and can be computed from the current one-time signature or verification key as mentioned in Sect.3.1 for Step 3 of Algorithm 3. If $s$ is odd, it is a right leaf thus LEAFCALC is not executed directly. To determine if $s$ is computed in treehash instance $\mathrm{TREEHASH}_{h}, h=0, \ldots, H-K-2$, we have to consider that in half of all cases it is copied and not computed. For this purpose we construct function $\delta_{H, K}^{\prime}(s)$ that returns the number of times leaf $s$ is computed in treehash

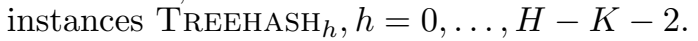

$$
\delta_{H, K}^{\prime}(s)=\sum_{h=0}^{H-K-2}\left\lfloor\frac{s \bmod 2^{h+1}}{2^{h}}\right\rfloor \cdot\left\lceil\frac{\left\lfloor\frac{s}{5 \cdot 2^{h}}\right\rfloor}{2^{H}}\right\rceil \cdot\left(1-\left\lfloor\frac{s \bmod 2^{h+2}}{2^{h+1}}\right\rfloor\right)
$$

The topmost treehash instance TreEHASH $H-K-1$ cannot copy nodes from the Rightnodes array because the required nodes have not been computed so far. Thus, we have to count the number of computations for this instance as in the unoptimized version. The total number of times leaf $s$ is generated during the computation of all authentication nodes can now be summed up to

$$
N_{H, K}^{\prime}(s)=\left\lfloor\frac{s \bmod 2^{H-K}}{2^{H-K-1}}\right\rfloor \cdot\left\lceil\frac{\left\lfloor\frac{s}{5 \cdot 2^{H-K-1}}\right\rfloor}{2^{H}}\right\rceil+\delta_{H, K}^{\prime}(s) .
$$

On average each leaf is now computed $\overline{N_{H, K}^{\prime}}=N_{H, K_{\text {total }}}^{\prime} / 2^{H} \approx \frac{1}{4}(H-K+1)$ times. The reduced number of computations for each leaf is shown in Fig. 2. Visual comparison between Figs. 1 and 2 already gives an intuition of the reduction and balancing of leaf computations. For further comparisons see Fig. 3 in the appendix. Table 2 compares the total number of leaf computations, how often a leaf has to be computed in the worst-case, and the average number of leaf computations for common heights $H=\{10,16,20\}$ and $K=\{2,4\}$. The total number of leaf 


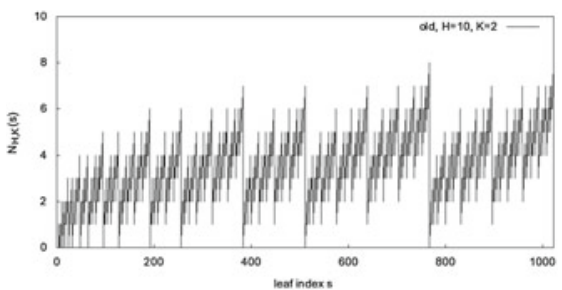

Fig. 1. Number of times each leaf is computed by the original BDS algorithm for a Merkle tree of height $H=$ 10 and $K=2$.

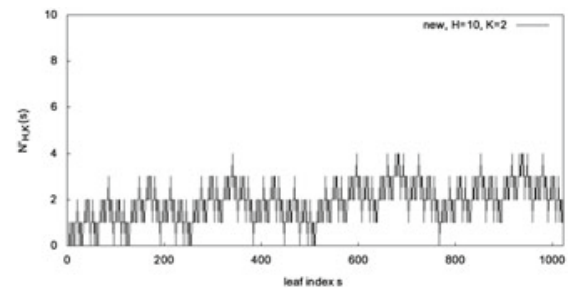

Fig. 2. Number of times each leaf is computed by our variation for a Merkle tree of height $H=10$ and $K=2$.

computations as well as the average computations per leaf are decreased by about 38-48\% for the chosen parameters of $H$ and $K$. Both the worst-case computation time as well as the average signature computation time are decreased. E.g., battery-powered devices greatly profit from the reduced overall computation time which directly relates to the overall power consumption.

Table 2. Overview of the necessary computations for a Merkle tree with parameters $H$ and $K$ when executing Algorithm 3. Furthermore, the worst-case computations for a leaf is listed together with the average computations $\overline{N_{H, K}}$ and $\overline{N_{H, K}^{\prime}}$. The variance of $N_{H, K}(s)$ and $N_{H, K}^{\prime}(s)$ is denoted by $\sigma_{H, K}^{2}$ and $\sigma_{H, K}^{\prime 2}$.

\begin{tabular}{lrrrrrrrrrrrrr}
\hline $\mathrm{H}$ & $\mathrm{K}$ & $N_{H, K_{\text {tot }}}$ & $N_{H, K_{\text {tot }}}^{\prime}$ & $N_{H, K}$ & \multicolumn{1}{c}{$N_{H, K}^{\prime}$} & $\%$ & $\sigma_{H, K}^{2}$ & $\sigma_{H, K}^{\prime 2}$ & $\%$ & $N_{H, K}(s)$ & $N_{H, K}^{\prime}(s)$ & $\%$ \\
\hline 10 & 2 & 3586 & 1921 & 3.50 & 1.88 & 46.4 & 2.24 & 0.73 & 67.3 & 8 & 4 & 50.0 \\
10 & 4 & 2946 & 1697 & 2.88 & 1.66 & 42.4 & 1.60 & 0.50 & 68.5 & 6 & 3 & 50.0 \\
10 & 6 & 2018 & 1257 & 1.97 & 1.23 & 37.7 & 1.02 & 0.33 & 67.9 & 4 & 2 & 50.0 \\
16 & 2 & 425986 & 221185 & 6.50 & 3.38 & 48.1 & 3.75 & 1.11 & 70.4 & 14 & 7 & 50.0 \\
16 & 4 & 385026 & 206849 & 5.88 & 3.16 & 46.3 & 3.11 & 0.88 & 71.6 & 12 & 6 & 50.0 \\
16 & 6 & 325634 & 178689 & 4.97 & 2.73 & 45.1 & 2.53 & 0.71 & 72.1 & 10 & 5 & 50.0 \\
20 & 2 & 8912898 & 4587521 & 8.50 & 4.38 & 48.5 & 4.75 & 1.36 & 71.4 & 18 & 9 & 50.0 \\
20 & 4 & 8257538 & 4358145 & 7.88 & 4.16 & 47.2 & 4.11 & 1.13 & 72.5 & 16 & 8 & 50.0 \\
20 & 6 & 7307266 & 3907585 & 6.97 & 3.73 & 46.5 & 3.53 & 0.96 & 72.9 & 14 & 7 & 50.0 \\
\hline
\end{tabular}

Since all but the topmost treehash instance only need to be computed every second time, the number of updates per signature (Algorithm 3, Step 5) can be reduced from $\lceil(H-K) / 2\rceil$ to $\lceil(H-K+1) / 4\rceil$. As a result, the average update time is much better balanced than in Algorithm 3 and the worst case computation time is also improved. The BDS algorithm needs to store $3 H+$ $\lfloor H / 2\rfloor-3 K+2^{K}-2$ tree nodes and $2(H-K)+1$ PRNG seeds as signature key. Due to storing the rightmost nodes our improved algorithm increases the 

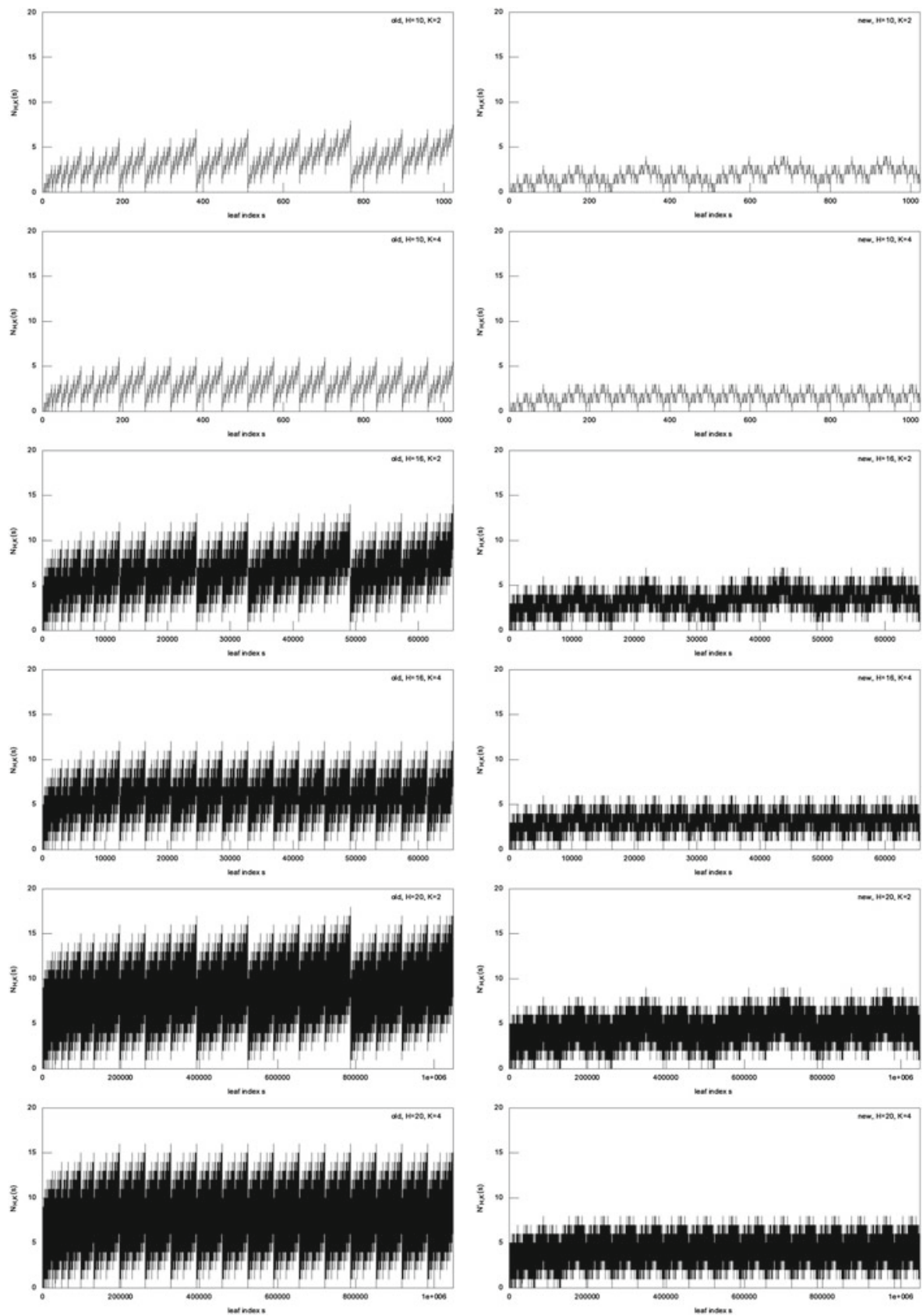

Fig. 3. Comparison of $N_{H, K}(s)$ and $N_{H, K}^{\prime}(s)$ for $H=\{10,16,20\}$ and $K=\{2,4\}$ for all leaves $s$ of the respective tree. 
number of tree nodes that have to be stored by $\left(\begin{array}{c}H-K \\ 2\end{array}\right)$. Even if the additional memory is used to increase $K$ for the original BDS algorithm, the speedup is still significant. E.g., comparing our $(H, K)=(16,4)$ to $\operatorname{BDS}(16,6)$ gives comparable storage requirements, but still a speedup of $36 \%$. The verification key and signature sizes remain unaffected: the verification key size is $m$ and the signature size remains at $t \cdot n+H \cdot m$.

\section{Implementation and Results}

In the following we describe our choices for the cryptographic primitives which we use to implement the proposed signature scheme described in Sects. 2 and 3. We then detail on the target platforms and give performance figures for key and signature generation as well as signature verification.

\subsection{A Bounded Leakage Merkle Signature Engine}

We implemented two versions with different hash functions $g$ for the Merkle tree. Both versions use AES-128 in an MJH construction [16]. Using AES-128 as block cipher is favorable from a performance perspective as existing AES coprocessors can be used. MJH is collision resistant for up to $\mathcal{O}\left(2^{\frac{2 n}{3}-\log n}\right)$ queries when instantiated with a $n$-bit block cipher. With AES-128 as an ideal cipher, this results in 80 bits security [16]. On the downside, MJH produces 256-bit hash outputs which in the MSS setting leads to an increased key and signature size. Hence, we also implement a version that shortens the 256-bit output of MJH to 160 -bit, resulting in smaller key and signature sizes. This also reduces the number of times the AES-engine needs to be called when creating nodes in the Merkle tree. Leakage of $g$ is not an issue, since $g$ only processes public information.

One-way function $f$ is implemented based on AES-128 in an MMO $[17,18]$ construction: $f\left(x_{i}\right):=\operatorname{AES}_{\mathrm{IV}}\left(x_{i}\right) \oplus x_{i}$. Unlike the PRNG, $f$ is keyless. Hence, for independent inputs its leakage is inherently 1-limiting and $f$ can thus be viewed as uniformly seed-preserving. The $P R N G$ defined in (1) is implemented based on the leakage-2-limiting PRNG proposed in [24]. In particular, $\operatorname{PRNG}\left(k_{i}\right):=$ $\left(\operatorname{AES}_{k_{i}}\left(0^{128}\right), \operatorname{AES}_{k_{i}}\left(0^{127} \| 1\right)\right)$, where $\operatorname{AES}_{k_{i}}$ denotes the AES-128 with a 128-bit key $k_{i}$, used as seed-preserving function.

Both PRNG and $f$ handle secret inputs. The PRNG processes each SEED $s$ and $\mathrm{SEEDW}_{\mathrm{W}-\mathrm{OTS}}$ as well as the $x_{i}$ for $s$ exactly $N_{H, K}^{\prime}(s)$ times during state updates and one time during signing $\mathrm{OTS}_{s}$. We exclude the key generation in this analysis, as it is performed off-chip, assumably in a secure environment. Both PRNG and $f$ rely on AES-128 as cryptographic building block. The PRNG executes AES twice under the same secret key (i.e. the PRNG is 2-limiting), while $f$ touches the secret input only once, making the signature engine overall leakage-2-limited. The strongest leakage will be observed for the $\mathrm{SEED}_{i}$, resulting in a total of $l=2 \cdot\left(\max \left(N_{H, K}^{\prime}(s)\right)+1\right)$ leakages. These $l$ observations are on 2 different inputs, i.e., there are $l / 2=\max \left(N_{H, K}^{\prime}(s)\right)+1$ observations under the same input (i.e., leakage will only differ by noise). Classical side-channel 
attacks are further mitigated by the fact that intermediate values $\operatorname{SEED}_{i}$ of the key generation PRNG are not output. The adversary will only get access to a limited number of $x_{i}$.

\subsection{Implementation Platforms}

We implement the signature scheme on two different platforms. On the one side we choose a lightweight and low-cost 8-bit Atmel ATxmega microcontroller and on the other side a powerful Intel Core i7 notebook CPU.

Intel Core i7-2620M 64-bit CPU. Intel's off-the-shelf Core i7-2620M 64bit Sandy Bridge notebook CPU [12] features two cores running at $2.70 \mathrm{GHz}$ (with Turbo Boost technology up to $3.40 \mathrm{GHz}$ ). For accurate measurement, we disabled Turbo Boost and hyper-threading during our benchmarks. The CPU incorporates the recent extensions to the $\mathrm{x} 86$ instruction set that improve the performance when en-/decrypting data using AES. The extension is called AESNI and consists of six additional instructions [13]. All standardized key lengths (128 bit, 192 bit, 256 bit) are supported for a block size of 128 bit.

Atmel AVR ATxmega128A1 8-bit Microcontroller. We are using the Atmel evaluation board AVR XPLAIN [3] that features an ATxmega128A1 microcontroller [1,2]. The ATxmega offers hardware accelerators for DES and AES and is clocked at $32 \mathrm{MHz}$. The hardware acceleration is limited to AES with 128-bit key and block size. A leakage analysis has been performed on this processor in Sect.4.4, as it is a typical example for a low-power embedded platform.

\subsection{Performance Results}

In the following we give performance figures of the signature scheme for selected Merkle tree heights $H$ and parameters $K$ and $w$ on both platforms.

CPU Performance. On the Intel CPU we measure the time it takes to create the root node of the Merkle tree, i.e., the verification key generation. We iterate over all leaves and sign random messages to measure the average computation time that is needed to create a valid MSS signature. Additionally, we measure the time it takes to verify an MSS signature. Signature computation includes creating the signing key, performing a one-time signature with the created signing key, and generating the next authentication path (the last step can be removed, as it can be precomputed at any time between two signing operations). The measurement is done for tree height $H=16$ with $K=2$ and $w=2$. Note, due to the binary tree structure the root node computation can be parallelized if more than one CPU core is available, which would bring down the required 
Table 3. Performance figures of a Merkle tree with parameters $H=16, K=2, w=2$ on an Intel i7 CPU and $H=10, K=2, w=2$ on an ATxmega microcontroller. $f$ is implemented using a hardware-accelerated AES-128 (AES-NI instructions, ATxmega crypto accelerator) in MMO construction. $g$ is implemented using AES-128 in an MJH256 construction and with the output truncated to $160 \mathrm{bit}$. The Intel CPU was clocked at $2.7 \mathrm{GHz}$ and the ATxmega at $32 \mathrm{MHz}$.

\begin{tabular}{|c|c|c|c|c|c|c|}
\hline \multirow{2}{*}{$\begin{array}{c}\text { Hash } g \\
\text { Target }\end{array}$} & \multicolumn{3}{|c|}{ MJH-256 w/ AES-128 } & \multicolumn{3}{|c|}{ MJH-160 w/ AES-128 } \\
\hline & {$[22]$} & our & Impr. (\%) & {$[22]$} & our & Impr. (\%) \\
\hline Core i7 KEYGEN & $6546.9 \mathrm{~ms}$ & $6037.5 \mathrm{~ms}$ & 8 & $4218.7 \mathrm{~ms}$ & $3886.3 \mathrm{~ms}$ & 8 \\
\hline Core i7 SIGN & 743.9 us & 401.3 us & 46 & $487.1 \mathrm{us}$ & 256.2 us & 47 \\
\hline Core i7 VERIFy & 76.1 us & 78.1 us & -3 & 50.8 us & 49.3 us & 3 \\
\hline Sign & $110.0 \mathrm{~ms}$ & $64.9 \mathrm{~ms}$ & 41 & $70.7 \mathrm{~ms}$ & $41.7 \mathrm{~ms}$ & 41 \\
\hline VERIFY & $18.4 \mathrm{~ms}$ & $18.4 \mathrm{~ms}$ & 0 & $11.0 \mathrm{~ms}$ & $11.0 \mathrm{~ms}$ & 0 \\
\hline
\end{tabular}

computation time by roughly the factor of cores used. We compare our results against the originally proposed signature scheme [22] in Table 3.

Compared to the previous results of [22] our improved algorithm in combination with the exchanged PRNG yields on average a performance gain of 46-47\% for signature generation. The new PRNG improves the computation time on average by $8 \%$, the algorithmic changes to the authentication path computation algorithm yield $38-39 \%$ points.

When generating verification keys an $8 \%$ improvement can be observed. This is due to the exchanged PRNG which uses a hardware-accelerated AES-engine since our algorithmic improvements do not affect key generation. Signature verification is more or less stable, regardless of cipher/algorithm combinations and is about a factor of 5 faster than signature generation.

Microcontroller Performance. On the microcontroller we measure the average computation time that is needed to create a valid MSS signature (including next authentication path computation) and the time it takes to verify an MSS signature. We omit the verification key generation since for reasonable tree heights it is an infeasible task for the microcontroller. Verification keys have to be computed once on a computer platform when initializing the microcontroller. The code was compiled using avr-gcc version 3.3.0. We found optimization stage -02 to provide the best tradeoff between runtime and code size.

The results on the microcontroller are in accordance with the results observed on the Intel CPU. The average signature generation time improves by $41 \%$ when using our proposed changes. Signature verification remains stable and is four times faster than signature generation. The memory consumption is listed in Table 4. Compared to the setting of [22] we need more flash and SRAM memory due to the additional storage for the RIGHTNODES array.

Table 5 compares key and signature sizes for different MSS implementations. Note that the increased signature sizes for [11] enable on-card key generation. 
Table 4. Required memory on the ATxmega128A1 microcontroller. In total $128 \mathrm{kByte}$ flash memory and $8 \mathrm{kByte}$ SRAM are available on this device. Memory consumption is reported in bytes and includes the verification and signature keys.

\begin{tabular}{|c|c|c|c|c|c|c|c|c|c|}
\hline \multirow[b]{3}{*}{$\mathrm{H}$} & \multirow[b]{3}{*}{ K } & \multicolumn{4}{|c|}{ MJH-256 w/ AES-128 } & \multicolumn{4}{|c|}{ MJH-160 w/ AES-128 } \\
\hline & & {$[22]$} & & our & & $\overline{[22]}$ & & our & \\
\hline & & Flash & SRAM & Flash & SRAM & Flash & $\overline{\text { SRAM }}$ & Flash & SRAM \\
\hline$\overline{10}$ & 2 & 10,608 & 1,486 & 12,070 & 2,382 & 10,204 & 1,066 & 11,352 & 1,626 \\
\hline 10 & 4 & 10,726 & 1,604 & 11,768 & 2,084 & 10,250 & 1,112 & 11,138 & 1,412 \\
\hline 10 & 6 & 11,994 & 2,874 & 12,752 & 3,066 & 11,018 & 1,878 & 11,726 & 1,998 \\
\hline
\end{tabular}

Table 5. Comparison of signing key (sk), verification key (vk), and signature size (sig) between [22], our improvement, and $\mathrm{XMSS}^{+}[11]$ for common $(H, K, w)$ parameter sets. All sizes are reported in bytes.

\begin{tabular}{|c|c|c|c|c|c|c|c|c|c|c|c|c|c|c|c|c|c|}
\hline \multirow[b]{2}{*}{$\mathrm{H}$} & \multirow[b]{2}{*}{$\mathrm{I} \quad \mathrm{K}$} & \multirow[b]{2}{*}{$\mathrm{w}$} & \multicolumn{3}{|c|}{ MJH-256 } & \multicolumn{3}{|c|}{ MJH-160 } & \multicolumn{3}{|c|}{ [22] (MJH-256) } & \multicolumn{3}{|c|}{ [22] (MJH-160) } & \multicolumn{3}{|c|}{$\mathrm{XMSS}^{+}[11]$} \\
\hline & & & sk & $\mathrm{vk}$ & sig & sk & $\mathrm{vk}$ & sig & sk & $\mathrm{vk}$ & sig & sk & $\mathrm{vk}$ & sig & $\overline{s k}$ & $\mathrm{vk}$ & sig \\
\hline 16 & 2 & 2 & 5,335 & 32 & 2,640 & 3,547 & 20 & 1,680 & 2,423 & 32 & 2,640 & 1,727 & 20 & 1,680 & 3,760 & 544 & 3,476 \\
\hline 16 & 2 & & 5,335 & 32 & 1,584 & 3,547 & 20 & 1,008 & 2,423 & 32 & 1,584 & 1,727 & 20 & 1,008 & 3,200 & 512 & 1,892 \\
\hline 20 & 4 & 2 & 7,049 & 32 & 2,768 & 4,649 & 20 & 1,760 & 3,209 & 32 & 2,768 & 2,249 & 920 & 1,760 & 4,303 & 608 & 3,540 \\
\hline 20 & 4 & 4 & 7,049 & 32 & 1,712 & 4,649 & 20 & 1,088 & 3,209 & 32 & 1,712 & 2,249 & 20 & 1,088 & 3,744 & 576 & 1,956 \\
\hline
\end{tabular}

\subsection{Leakage Results}

The leakage of the AVR ATxmega processors with respect to power analysis has been analyzed in [14]. The found leakage is weak: the best attack needs more than 3000 measurements on random known inputs to recover the secret key. However, the applied method is not the most powerful ${ }^{1}$.

In order to get a more thorough leakage analysis of the target platform, we performed own side-channel experiments. Since all AES computations with critical leakage are performed by the AES co-processor of the ATxmega processor [2], we analyzed the leakage of that co-processor. Instead of a correlation based DPA, we applied a (univariate) template attack [7], the de-facto standard for power leakage evaluation [23]. The profiled intermediate state is $\Delta=p_{0} \oplus k_{0} \oplus p_{1} \oplus k_{1}$, where one template was created for each possible $\Delta$. This is the same intermediate state that was targeted in [14]. It appears to be the intermediate state with the strongest leakage. Each recovered $\Delta$ reveals one byte of key information. The maximum observable leakage is that of the 2-limiting PRNG, which is, at most, executed 10 times each on two different inputs (for $(H, K)=(20,2)$ ). To capture this maximal leakage, the experiment builds univariate templates from 10,000 traces and tests over two groups of 10 traces (each group shares the same input). A total of 5000 experiments are conducted, resulting in a Guessing Entropy [23] of 85.06 or 6.41 bits for the correct $\Delta$. This means that the adversary still has

\footnotetext{
${ }^{1}$ Both targeting the key xor and using correlation attack are not considered optimal methods of leakage extraction.
} 
to test more than 85 hypotheses for that byte on average. The reduction in entropy is hence less than $0.6 \mathrm{bit}^{2}$, resulting in well above $100 \mathrm{bit}$ of remaining key entropy when considering univariate side-channel attacks.

An alternative to plain template attacks are algebraic side-channel attacks [21], which do not require known input and output and would be more applicable to attack the PRNG in this work. While being able to exploit several (close to 1000 in [21]) leakages during a single execution of AES, these methods are very sensitive to noise and need a much stronger leakage than the one observed here. Often, an almost noise-free Hamming weight leakage is assumed, which is more than $2.5 \mathrm{bits}$ of information on a byte. This kind of information is not provided by the observed leakage of the hardware AES of the ATxmega processor.

The remaining point of attack is in the Winternitz signature, where the adversary actually gets access to hash outputs and some outputs of the PRNG used to generate the one-time keys. The observed leakage (10 observations for the same single input, same setup as for the PRNG) has a guessing entropy of 99.53 , i.e. less than 0.4 bit of information per byte are revealed. Not much prior work on side-channel attacks on one-way functions has been performed which is most likely due to the fact that the adversary gets only single observations of the leakage.

\section{Conclusion}

We presented a novel algorithmic improvement for authentication path computation in MSS that balances leaf computations and reduces side-channel leakage. The proposed improvements have been implemented on two platforms and were compared to previous proposed algorithms showing significant improvements. Furthermore, we gave explicit formulas to quantify the number of leaf computations when using MSS and showed that the leakage of the secret state is bounded throughout the entire scheme. The leakage analysis of the ATxmega AES engine showed that no significant information can be extracted about the secret state, due to the bounded number of executions under the same key.

We stated theoretically achievable performance gains and verified them practically. The algorithmic improvement decreases the required computation time for signature creation in theory as well as in practice. The performance figures show that Merkle signatures are not only practical, but also resource-friendly and fast and have inherently bounded side-channel leakage. As such they are a advantageous choice for, e.g., digital signature smartcards.

Acknowledgments. This material is based in part upon work supported by the National Science Foundation under Grant No. 1261399 and by grant 01ME12025 SecMobil of the German Federal Ministry of Economics and Technology. We would like to thank the anonymous reviewers for their helpful comments.

\footnotetext{
${ }^{2}$ Note that the guessing entropy for a byte with $2^{8}$ equiprobable states is 128 , i.e. 7 bits as guessing entropy looks for the expected number of guesses.
} 


\section{A Appendix}
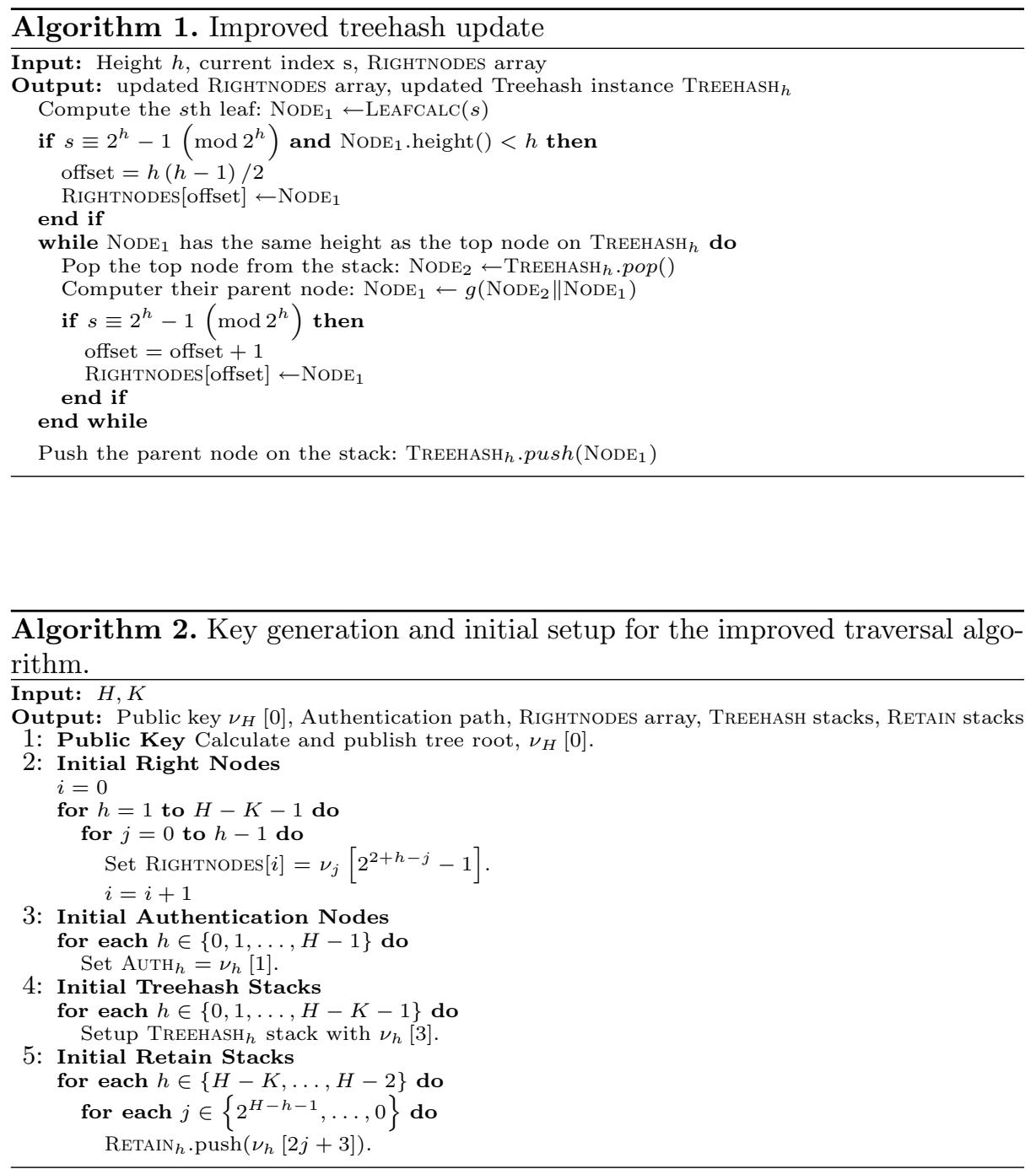


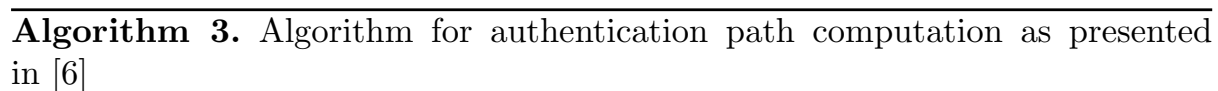

Input: $s \in\left\{0, \ldots, 2^{H}-2\right\}, H, K$, and the algorithm state.

Output: Authentication path $A_{s+1}$ for leaf $s+1$.

1: Let $\tau=0$ if leaf $s$ is a left node or let $\tau$ be the height of the first parent of leaf $s$ which is a left node: $\tau \leftarrow \max \left\{h: 2^{h} \mid(s+1)\right\}$

2: If the parent of leaf $s$ on height $\tau+1$ is a left node, store the current authentication node on height $\tau$ in $\operatorname{KeEP}_{\tau}$ :

if $\left\lfloor s / 2^{\tau+1}\right\rfloor$ is even and $\tau<H-1$ then $\mathrm{KEEP}_{\tau} \leftarrow \mathrm{AUTH}_{\tau}$

3: If leaf $s$ is a left node, it is required for the authentication path of leaf $s+1$ :

if $\tau=0$ then $\operatorname{Auth}_{0} \leftarrow \operatorname{LeAfcalC}(s)$

4: Otherwise, if leaf $s$ is a right node, the auth. path for leaf $s+1$ changes on heights $0, \ldots, \tau$ :

if $\tau>0$ then

a) The authentication path for leaf $s+1$ requires a new left node on height

$\tau$. It is computed using the current authentication node on height $\tau-1$

and the node on height $\tau-1$ previously stored in $\operatorname{KeEP}_{\tau-1}$. The node

stored in $\operatorname{KEEP}_{\tau-1}$ can then be removed:

$\mathrm{AUTH}_{\tau} \leftarrow g\left(\mathrm{AUTH}_{\tau-1} \| \mathrm{KEEP}_{\tau-1}\right)$, remove $\mathrm{KeEP}_{\tau-1}$

b) The authentication path for leaf $s+1$ requires new right nodes on heights

$h=0, \ldots, \tau-1$. For $h<H-K$ these nodes are stored in TreEHASH $_{h}$

and for $h \geq H-K$ in $\operatorname{RETAIN}_{h}$ :

for $h=0$ to $\tau-1$ do

if $h<H-K$ then Auth $_{h} \leftarrow$ TreEHaSh $_{h} \cdot \operatorname{pop}()$

if $h \geq H-K$ then $\mathrm{AuTH}_{h} \leftarrow \operatorname{RETAIN}_{h} \cdot \operatorname{pop}()$

c) For heights $0, \ldots, \min \{\tau-1, H-K-1\}$ the Treehash instances must be initialized anew. The Treehash instance on height $h$ is initialized with the start index $s+1+3 \cdot 2^{h}<2^{H}$ :

for $h=0$ to $\min \{\tau-1, H-K-1\}$ do

TreEHASH $_{h} \cdot \operatorname{initialize}\left(s+1+3 \cdot 2^{h}\right)$

5: Next we spend the budget of $(H-K) / 2$ updates on the Treehash instances to prepare upcoming authentication nodes:

repeat $(H-K) / 2$ times

a) We consider only stacks which are initialized and not finished. Let $k$ be the index of the Treehash instance whose lowest tail node has the lowest height. In case there is more than one such instance we choose the instance with the lowest index:

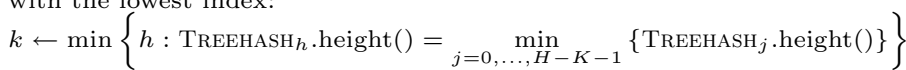

b) The Treehash instance with index $k$ receives one update: TrEeHASH $_{k} \cdot$ update()

6: The last step is to output the authentication path for leaf $s+1$ : return $\mathrm{Auth}_{0}, \ldots, \mathrm{Auth}_{H-1}$.

\section{References}

1. Atmel. ATxmega128A1 Data Sheet. http://www.atmel.com/dyn/resources/prod_ documents/doc8067.pdf

2. Atmel. AVR XMEGA A Manual. http://www.atmel.com/dyn/resources/prod_ documents/doc8077.pdf

3. Atmel. AVR XPLAIN board. http://www.atmel.com/dyn/resources/prod_ documents/doc8203.pdf

4. Buchmann, J., Dahmen, E., Ereth, S., Hülsing, A., Rückert, M.: On the security of the winternitz one-time signature scheme. In: Nitaj, A., Pointcheval, D. (eds.) Progress in Cryptology AFRICACRYPT 2011. LNCS, vol. 6737, pp. 363378. Springer, Berlin / Heidelberg (2011)

5. Buchmann, J., Dahmen, E., Hülsing, A.: XMSS - a practical forward secure signature scheme based on minimal security assumptions. In: Yang, B.-Y. (ed.) PQCrypto 2011. LNCS, vol. 7071, pp. 117-129. Springer, Heidelberg (2011) 
6. Buchmann, J., Dahmen, E., Szydlo, M.: Hash-based digital signature schemes. In: Bernstein, D.J., Buchmann, J., Dahmen, E. (eds.) Post-Quantum Cryptography, pp. 35-93. Springer, Heidelberg (2009)

7. Chari, S., Rao, J.R., Rohatgi, P.: Template Attacks. In: Kaliski, B.S., Koç, çK, Paar, C. (eds.) CHES 2002. LNCS, vol. 2523, pp. 13-28. Springer, Heidelberg (2003)

8. Dods, C., Smart, N.P., Stam, M.: Hash based digital signature schemes. In: Smart, N.P. (ed.) Cryptography and Coding 2005. LNCS, vol. 3796, pp. 96-115. Springer, Heidelberg (2005)

9. Faust, S., Kiltz, E., Pietrzak, K., Rothblum, G.N.: Leakage-resilient signatures. In: Micciancio, D. (ed.) TCC 2010. LNCS, vol. 5978, pp. 343-360. Springer, Heidelberg (2010)

10. Hülsing, A.: W-OTS+ - shorter signatures for hash-based signature schemes. In: Youssef, A., Nitaj, A., Hassanien, A.E. (eds.) AFRICACRYPT 2013. LNCS, vol. 7918, pp. 173-188. Springer, Heidelberg (2013)

11. Hülsing, A., Busold, C., Buchmann, J.: Forward secure signatures on smart cards. In: Knudsen, L.R., Wu, H. (eds.) SAC 2012. LNCS, vol. 7707, pp. 66-80. Springer, Heidelberg (2013)

12. Intel. Intel Core i7 2620M Specifications. http://ark.intel.com/products/52231/ Intel-Core-i7-2620M-Processor-(4M-Cache-2_70-GHz)

13. Intel. Whitepaper on the Intel AES Instructions Set. http://software.intel.com/ file/24917

14. Kizhvatov, I.: Side channel analysis of AVR XMEGA crypto engine. In: Proceedings of the 4th Workshop on Embedded Systems Security, WESS '09, pp. 8:1-8:7. ACM (2009)

15. Lamport, L.: Constructing digital signatures from a one-way function. Technical report, CSL-98, SRI, International (1979)

16. Lee, J., Stam, M.: MJH: a faster alternative to MDC-2. In: Kiayias, A. (ed.) Topics in Cryptology CT-RSA 2011. LNCS, vol. 6558, pp. 213-236. Springer, Berlin / Heidelberg (2011)

17. Matyas, S.M., Meyer, C.H., Oseas, J.: Generating strong one-way functions with cryptographic algorithm. IBM Tech. Discl. Bull. 27(10A), 5658-5659 (1985)

18. Menezes, A., Van Oorschot, P., Vanstone, S.: Handbook of Applied Cryptography. CRC Press, Boca Raton (1997). Algorithm 9.41

19. Merkle, R.C.: A certified digital signature. In: Brassard, G. (ed.) CRYPTO 1989. LNCS, vol. 435, pp. 218-238. Springer, Heidelberg (1990)

20. Micali, S., Reyzin, L.: Physically observable cryptography. In: Naor, M. (ed.) TCC 2004. LNCS, vol. 2951, pp. 278-296. Springer, Heidelberg (2004)

21. Renauld, M., Standaert, F.-X., Veyrat-Charvillon, N.: Algebraic side-channel attacks on the AES: why time also matters in DPA. In: Clavier, C., Gaj, K. (eds.) CHES 2009. LNCS, vol. 5747, pp. 97-111. Springer, Heidelberg (2009)

22. Rohde, S., Eisenbarth, T., Dahmen, E., Buchmann, J., Paar, C.: Fast hashbased signatures on constrained devices. In: Grimaud, G., Standaert, F.-X. (eds.) CARDIS 2008. LNCS, vol. 5189, pp. 104-117. Springer, Heidelberg (2008)

23. Standaert, F.-X., Malkin, T.G., Yung, M.: A unified framework for the analysis of side-channel key recovery attacks. In: Joux, A. (ed.) EUROCRYPT 2009. LNCS, vol. 5479, pp. 443-461. Springer, Heidelberg (2009) 
24. Standaert, F.-X., Pereira, O., Yu, Y., Quisquater, J.-J., Yung, M., Oswald, E.: Leakage resilient cryptography in practice. In: Sadeghi, A.-R., Naccache, D., Basin, D., Maurer, U. (eds.) Towards Hardware-Intrinsic Security. Information Security and Cryptography, pp. 99-134. Springer, Heidelberg (2010)

25. Szydlo, M.: Merkle tree traversal in log space and time. In: Cachin, C., Camenisch, J.L. (eds.) EUROCRYPT 2004. LNCS, vol. 3027, pp. 541-554. Springer, Heidelberg (2004) 